\title{
DNA Relatedness among Verticil-Forming Streptomyces Species (Formerly Streptoverticillium Species)
}

\author{
DAVID P. LABEDA* \\ Microbial Properties Research, National Center for Agricultural Utilization Research, Agricultural \\ Research Service, U.S. Department of Agriculture, Peoria, Illinois 61604
}

\begin{abstract}
Levels of DNA relatedness among 35 strains of Streptomyces species originally classified in the genus Streptoverticillium were determined spectrophotometrically. These strains represent eight of the phenotypic cluster groups described for the genus Streptoverticillium in Bergey's Manual of Systematic Bacteriology. Average linkage clustering of the DNA relatedness data resulted in 20 clusters including 13 single-member clusters, at a level of relatedness of $>70 \%$. Several species could be reduced to synonymy on the basis of DNA homology data, but these taxa were not generally equivalent to the clusters suggested by phenotypic numerical taxonomy data.
\end{abstract}

The genus Streptoverticillium was described by Baldacci (1) to encompass strains which were previously described as members of the genus Streptomyces but had the following morphological property: production of long, straight hyphae containing side branches arranged in verticils bearing terminal umbels of twisted sporogenous hyphae. The taxonomy of the genus Streptoverticillium has been a subject of discussion for a long time, and the number of species in this genus has ranged from 2, as proposed by Pridham (11), to the 66 nomenspecies grouped by Locci and Schofield (8) in 10 major clusters and 14 single-member clusters on the basis of the results of the numerical taxonomic study of Locci et al. (7).

There is phenetic overlap between the streptoverticillia and Streptomyces strains, particularly members of the Streptomyces lavendulae cluster, but the results of previous rRNA-DNA hybridization studies (2) and differences in specific physiological properties supported the separation of the genus Streptoverticillium from the genus Streptomyces. Recently, however, Witt and Stackebrandt (18) proposed, on the basis of the results of an analysis of 16S rRNA sequences of strains of streptoverticillia and streptomycetes, that the two genera should be unified because of the dispersion of the streptoverticillia among the streptomycetes in their phylogenetic analysis.

An analysis of the DNA relatedness of the nomenspecies in and between the major phenotypic clusters delineated by Locci and Schofield (8) was performed to determine the genetic integrity of these clusters.

\section{MATERIALS AND METHODS}

Strains and cultivation conditions. The strains included in this study are shown in Table 1 . These strains represent eight of the phenotypic clusters of Locci and Schofield (8) and include the type strain of each nomenspecies as well as the type strains of other verticillate taxa that have not been validly described yet. The primary method of storage of the strains was as refrigerated ampoules containing freeze-dried spore and vegetative mycelium suspensions in sterile beef serum. Working stock cultures were maintained on slants of inorganic salts-starch agar or yeast extract-malt extract agar (15) and were stored at $4^{\circ} \mathrm{C}$ until they were needed. Biomass for extraction of DNA was grown in tryptoneglucose-liver extract-yeast extract broth (12) with shaking at $200 \mathrm{rpm}$ at $28^{\circ} \mathrm{C}$.

DNA isolation, purification, and hybridization. DNA was extracted from cells by the method of Marmur (10) after they were broken by passage through a French pressure cell (SLM Instruments, Inc., Urbana, Ill.) at $10,000 \mathrm{lb} / \mathrm{in}^{2}$. The DNA was purified by chromatography on hydroxyapatite or by cesium chloride density gradient centrifugation (9). The levels of DNA relatedness between strains were calculated from $C_{0} t_{0.5}$ values in $5 \times \mathrm{SSC}(1 \times \mathrm{SSC}$ is $0.15 \mathrm{M} \mathrm{NaCl}$ plus $0.015 \mathrm{M}$ sodium citrate) supplemented with $20 \%$ dimethyl sulfoxide at $65^{\circ} \mathrm{C}$

* Mailing address: National Center for Agricultural Utilization Research, 1815 North University Street, Peoria, IL 61604.
$\left(T_{m}-23^{\circ} \mathrm{C}\right)$ by the method of Seidler and Mandel (14) and Seidler et al. (13) as described in Kurtzman et al. (3).

Statistical calculations. Statistical calculations involving the DNA relatedness data were performed by using SAS for Windows, release 6.11 (SAS Institute Inc., Cary, N.C.), and a Zeos Pantera 120-MHz Pentium microcomputer (Zeos Information Systems, Minneapolis, Minn.). The data were clustered by the average-linkage algorithm using the SAS/STAT CLUSTER procedure, and a dendrogram was generated with SAS/GRAPH by using the macro GRAFTREE, which was written and kindly provided by Dan Jacobs, University of Maryland.

\section{RESULTS AND DISCUSSION}

The genus Streptoverticillium was described in Bergey's Manual of Systematic Bacteriology, vol. 4 (8), as a taxon that consists of 24 species (10 species groups and 14 single-member clusters), as determined by a numerical taxonomic analysis of 111 Streptoverticillium and Streptomyces strains with pseudoverticillate sporophores in which 185 morphological and physiological unit characters were examined. The levels of DNA relatedness for the 35 strains representing eight phenotypic clusters studied in this investigation support the hypothesis that there are 20 distinct species defined by levels of DNA relatedness equal to or greater than $70 \%$. In general, the clusters based on DNA relatedness data are not the same as the cluster groups formed on the basis of numerical taxonomic results. Similar observations were made in DNA relatedness studies involving strains belonging to the Streptomyces cyaneus (5), S. lavendulae (4), and Streptomyces violaceusniger (6) numerical taxonomic cluster groups defined by Williams et al. (17).

Some strains belonging to each phenotypic cluster do exhibit significant levels of chromosomal similarity (level of DNA relatedness, $>70 \%$ ), indicating that they represent synonymous species. This can be clearly seen in the DNA relatedness data in Table 2 and the dendrogram resulting from average clustering of these data (Fig. 1).

All of the strains belonging to the Streptomyces abikoensis phenotypic cluster evaluated in this study exhibited more than 81\% DNA relatedness to each other, indicating that the species names of these strains, "Streptomyces takataensis" NRRL B-12516 and Streptomyces waksmanii NRRL B-1707 ${ }^{\mathrm{T}}(\mathrm{T}=$ type strain), are truly subjective synonyms of $S$. abikoensis NRRL B-2113 ${ }^{\mathrm{T}}$. These data are in good agreement with data obtained in the previous DNA relatedness study of verticillate streptomycetes performed by Toyama et al. (16), although Toyama et al. obtained homology values of 70 to $72 \%$ by using the filter binding method. My data also indicate that "Streptomyces rubroverticillatus" NRRL B-16433 is misplaced in the Streptomyces baldaccii cluster, since it exhibits very high levels of DNA relatedness ( 81 to $100 \%$ ) to all of the strains in the $S$. 
TABLE 1. Verticillate Streptomyces (Streptoverticillium) species included in this study

\begin{tabular}{|c|c|c|}
\hline Cluster group $^{a}$ & Species & Strain $^{b}$ \\
\hline \multirow[t]{3}{*}{$\begin{array}{l}\text { Streptomyces abikoensis (formerly } \\
\text { Streptoverticillium abikoense) }\end{array}$} & Streptomyces abikoensis ${ }^{c}$ & $\begin{array}{l}\text { NRRL B-2113 }{ }^{\mathrm{T}}\left(=\text { ATCC } 12766^{\mathrm{T}}=\text { CBS } 487.62^{\mathrm{T}}=\text { DSM } 40831^{\mathrm{T}}=\text { IFO } 13860^{\mathrm{T}}\right. \\
\left.\quad=\mathrm{JCM} 4002^{\mathrm{T}}\right)\end{array}$ \\
\hline & "Streptomyces takataensis" & $\begin{array}{l}\text { NRRL B-12516 (= ATCC } 27469=\text { CBS } 771.62=\text { DSM } 40576=\text { IFO } 13476= \\
\text { JCM 4836) }\end{array}$ \\
\hline & Streptomyces waksmanii ${ }^{d}$ & $\begin{array}{l}\text { NRRL B-1707 }\left(=\text { ATCC } 12629^{\mathrm{T}}=\text { ATCC } 25494^{\mathrm{T}}=\text { CBS } 373.58^{\mathrm{T}}=\text { CBS }\right. \\
\left.954.69^{\mathrm{T}}=\text { DSM } 40464^{\mathrm{T}}=\text { IFO } 13082^{\mathrm{T}}=\mathrm{JCM} 4514^{\mathrm{T}}=\mathrm{JCM} 4548^{\mathrm{T}}\right)\end{array}$ \\
\hline \multirow[t]{12}{*}{$\begin{array}{l}\text { Streptomyces baldaccii (formerly } \\
\text { Streptoverticillium baldaccii) }\end{array}$} & Streptomyces baldaccii ${ }^{c}$ & $\begin{array}{l}\text { NRRL B-3500 }{ }^{\mathrm{T}}\left(=\text { ATCC } 23654^{\mathrm{T}}=\text { DSM } 40845^{\mathrm{T}}=\text { IFO } 14693^{\mathrm{T}}=\mathrm{JCM} 4272^{\mathrm{T}}\right. \\
\left.\quad=\text { IFO } 14693^{\mathrm{T}}\right)\end{array}$ \\
\hline & Streptomyces biverticillatus ${ }^{c}$ & NRRL ISP-5272 $\left(=\right.$ ATCC $23615^{\mathrm{T}}=$ CBS $668.68^{\mathrm{T}}=$ IFO $\left.12845^{\mathrm{T}}=\mathrm{JCM} 4431^{\mathrm{T}}\right)$ \\
\hline & Streptomyces diastallicus ${ }^{c}$ & NRRL $2886^{\mathrm{T}}\left(=\mathrm{DSM} 40846^{\mathrm{T}}=\mathrm{NCIB} 8936^{\mathrm{T}}=\mathrm{JCM} 4544^{\mathrm{T}}\right)$ \\
\hline & Streptomyces fervens ${ }^{c}$ & $\begin{array}{l}\text { NRRL } 2755^{\mathrm{T}}\left(=\text { ATCC } 27429^{\mathrm{T}}=\text { CBS } 644.72^{\mathrm{T}}=\text { DSM } 40086^{\mathrm{T}}=\text { IFO } 13343^{\mathrm{T}}=\right. \\
\left.\text { JCM } 4310^{\mathrm{T}}=\text { JCM } 4750^{\mathrm{T}}\right)\end{array}$ \\
\hline & Streptomyces flavopersicus ${ }^{c}$ & $\begin{array}{l}\text { NRRL } 2820^{\mathrm{T}}\left(=\text { ATCC } 19756^{\mathrm{T}}=\text { CBS } 494.68^{\mathrm{T}}=\text { DSM } 40093^{\mathrm{T}}=\text { IFO } 12769^{\mathrm{T}}=\right. \\
\left.\text { JCM } 4307^{\mathrm{T}}=\text { JCM } 4370^{\mathrm{T}}\right)\end{array}$ \\
\hline & Streptomyces griseoverticillatus ${ }^{c}$ & $\begin{array}{l}\text { NRRL B-12432 }\left(=\text { ATCC } 27436^{\mathrm{T}}=\text { CBS } 721.72^{\mathrm{T}}=\text { DSM } 40507^{\mathrm{T}}=\mathrm{IFO}\right. \\
\left.13420^{\mathrm{T}}=\text { RIA } 1381^{\mathrm{T}}=\mathrm{JCM} 4202^{\mathrm{T}}=\mathrm{JCM} 4767^{\mathrm{T}}\right)\end{array}$ \\
\hline & Streptomyces hiroshimensis ${ }^{c}$ & $\begin{array}{l}\text { NRRL B-5484 }\left(=\text { ATCC } 19772^{\mathrm{T}}=\text { CBS } 510.68^{\mathrm{T}}=\text { DSM } 40037^{\mathrm{T}}=\mathrm{IFO} 3839^{\mathrm{T}}\right. \\
\left.\quad=\text { IFO } 12785^{\mathrm{T}}=\mathrm{JCM} 4098^{\mathrm{T}}=\mathrm{JCM} 4586^{\mathrm{T}}=\text { NCIB } 9838^{\mathrm{T}}\right)\end{array}$ \\
\hline & Streptomyces kentuckensis ${ }^{c}$ & $\begin{array}{l}\text { NRRL B-1831 } 1^{\mathrm{T}}\left(=\text { ATCC } 12691^{\mathrm{T}}=\text { ATCC } 23926^{\mathrm{T}}=\text { CBS } 910.68^{\mathrm{T}}=\text { DSM }\right. \\
\left.40052^{\mathrm{T}}=\text { IFO } 12880^{\mathrm{T}}=\mathrm{JCM} 4153^{\mathrm{T}}=\mathrm{JCM} 4647^{\mathrm{T}}=\text { NCIB } 9624^{\mathrm{T}}\right)\end{array}$ \\
\hline & Streptomyces netropsis ${ }^{c}$ & $\begin{array}{l}\text { NRRL } 2268^{\mathrm{T}}\left(=\text { ATCC } 23940^{\mathrm{T}}=\text { CBS } 924.68^{\mathrm{T}}=\text { DSM } 40259^{\mathrm{T}}=\text { IFO } 12893^{\mathrm{T}}=\right. \\
\left.\text { JCM } 4063^{\mathrm{T}}=\text { JCM } 4655^{\mathrm{T}}\right)\end{array}$ \\
\hline & Streptomyces roseoverticillatus ${ }^{c}$ & $\begin{array}{l}\text { NRRL B-1993 }\left(=\text { ATCC } 19807^{\mathrm{T}}=\text { CBS } 560.68^{\mathrm{T}}=\text { DSM } 40039^{\mathrm{T}}=\text { IFO } 3844^{\mathrm{T}}\right. \\
\left.\quad=\text { IFO } 12817^{\mathrm{T}}\right)\end{array}$ \\
\hline & "Streptomyces rubrochlorinus" & NRRL B-12558 (= DSM $40850=$ IFO $14694=$ JCM 4281) \\
\hline & "Streptomyces rubroverticillatus" & NRRL B-16433 (= DSM $41489=$ JCM 4679) \\
\hline \multirow{5}{*}{$\begin{array}{l}\text { Streptomyces cinnamoneus subsp. } \\
\text { cinnamoneus (formerly Strepto- } \\
\text { verticillium cinnamoneum subsp. } \\
\text { cinnamoneum) }\end{array}$} & $\begin{array}{l}\text { Streptomyces cinnamoneus } \\
\text { subsp. cinnamoneus }{ }^{c}\end{array}$ & $\begin{array}{l}\text { NRRL B- } 1285^{\mathrm{T}}\left(=\text { ATCC } 11874^{\mathrm{T}}=\text { ATCC } 23897^{\mathrm{T}}=\text { CBS } 293.64^{\mathrm{T}}=\text { CBS }\right. \\
683.68^{\mathrm{T}}=\text { DSM } 40005^{\mathrm{T}}=\text { IFO } 12852^{\mathrm{T}}=\mathrm{JCM} 4152^{\mathrm{T}}=\mathrm{JCM} 4633^{\mathrm{T}}=\mathrm{NCIB} \\
\left.8851^{\mathrm{T}}\right)\end{array}$ \\
\hline & Streptomyces blastmyceticus $^{c}$ & $\begin{array}{l}\text { NRRL B-5480 } 0^{\mathrm{T}}\left(=\text { ATCC } 19731^{\mathrm{T}}=\text { CBS } 470.68^{\mathrm{T}}=\text { DSM } 40029^{\mathrm{T}}=\text { IFO } 12747^{\mathrm{T}}\right. \\
\left.\quad=\mathrm{JCM} 4184^{\mathrm{T}}=\mathrm{JCM} 4565^{\mathrm{T}}=\text { NCIB } 9800^{\mathrm{T}}\right)\end{array}$ \\
\hline & $\begin{array}{l}\text { Streptomyces cinnamoneus } \\
\text { subsp. albosporus }\end{array}$ & NRRL B-5624 $\left(=\right.$ ATCC $25186^{\mathrm{T}}=$ DSM $\left.40897^{\mathrm{T}}=\mathrm{JCM} 4968^{\mathrm{T}}\right)$ \\
\hline & Streptomyces eurocidicus ${ }^{c}$ & $\begin{array}{l}\text { NRRL B-1676 }{ }^{\mathrm{T}}\left(=\text { ATCC } 27428^{\mathrm{T}}=\text { CBS } 792.72^{\mathrm{T}}=\text { DSM } 40604^{\mathrm{T}}=\text { IFO } 13491^{\mathrm{T}}\right. \\
\left.=\text { JCM } 4029^{\mathrm{T}}=\mathrm{JCM} 4749^{\mathrm{T}}\right)\end{array}$ \\
\hline & Streptomyces lavenduligriseus ${ }^{c}$ & NRRL B-3173'T $\left(=\right.$ ATCC $13306^{\mathrm{T}}=$ CBS $\left.706.72^{\mathrm{T}}\right)$ \\
\hline \multirow{7}{*}{$\begin{array}{l}\text { Streptomyces griseocarneus } \\
\text { (formerly Streptoverticillium } \\
\text { griseocarneum) }\end{array}$} & Streptomyces griseocarneus ${ }^{c}$ & $\begin{array}{l}\text { NRRL B-1068 }\left(=\text { ATCC } 12628^{\mathrm{T}}=\text { ATCC } 19763^{\mathrm{T}}=\text { CBS } 501.68^{\mathrm{T}}=\mathrm{DSM}\right. \\
\left.40004^{\mathrm{T}}=\mathrm{IFO} 12776^{\mathrm{T}}=\mathrm{JCM} 4095^{\mathrm{T}}=\mathrm{JCM} 4580^{\mathrm{T}}\right)\end{array}$ \\
\hline & Streptomyces ardus ${ }^{d}$ & $\begin{array}{l}\text { NRRL } 2817^{\mathrm{T}}\left(=\text { ATCC } 27417^{\mathrm{T}}=\text { CBS } 731.72^{\mathrm{T}}=\operatorname{DSM} 40527^{\mathrm{T}}=\text { IFO } 13430^{\mathrm{T}}=\right. \\
\left.\text { JCM } 4543^{\mathrm{T}}=\text { JCM } 4722^{\mathrm{T}}\right)\end{array}$ \\
\hline & $\begin{array}{l}\text { Streptomyces cinnamoneus } \\
\text { subsp. azacoluta }\end{array}$ & NRRL B-1699 $\left(=\right.$ ATCC $12686^{\mathrm{T}}=$ CBS $\left.369.58^{\mathrm{T}}=\mathrm{IFO} 12363^{\mathrm{T}}=\mathrm{JCM} 4435^{\mathrm{T}}\right)$ \\
\hline & Streptomyces mashuensis ${ }^{c}$ & $\begin{array}{l}\text { NRRL B-8164 }\left(=\text { ATCC } 23934^{\mathrm{T}}=\text { CBS } 279.65^{\mathrm{T}}=\text { CBS } 918.68^{\mathrm{T}}=\text { DSM } 40221^{\mathrm{T}}\right. \\
\left.\quad=\text { IFO } 12888^{\mathrm{T}}=\mathrm{JCM} 4059^{\mathrm{T}}=\mathrm{JCM} 4650^{\mathrm{T}}\right)\end{array}$ \\
\hline & Streptomyces olivoverticillatus ${ }^{c}$ & $\begin{array}{l}\text { NRRL B-1994 }\left(=\text { ATCC } 25480^{\mathrm{T}}=\text { CBS } 890.69^{\mathrm{T}}=\text { DSM } 40250^{\mathrm{T}}=\text { IFO } 3842^{\mathrm{T}}\right. \\
\left.\quad=\text { IFO } 3929^{\mathrm{T}}=\text { IFO } 13068^{\mathrm{T}}=\mathrm{JCM} 4100^{\mathrm{T}}=\text { JCM } 4501^{\mathrm{T}}=\text { NCIB } 97148^{\mathrm{T}}\right)\end{array}$ \\
\hline & $\begin{array}{l}\text { "Streptomyces pentaticus subsp. } \\
\text { jenesis" }\end{array}$ & NRRL B-12088 ${ }^{\mathrm{T}}\left(=\mathrm{DSM} 40848^{\mathrm{T}}=\mathrm{JCM} 4211^{\mathrm{T}}\right)$ \\
\hline & "Streptomyces tropicalensis" & $\begin{array}{l}\text { NRRL B-12481 }(=\text { ATCC } 17963=\text { CBS } 729.72=\text { DSM } 40520=\text { IFO } 13428= \\
\text { JCM 4845) }\end{array}$ \\
\hline \multirow{2}{*}{$\begin{array}{l}\text { Streptomyces hachijoensis } \\
\text { (formerly Streptoverticillium } \\
\text { hachijoense) }\end{array}$} & Streptomyces hachijoensis ${ }^{c}$ & $\begin{array}{l}\text { NRRL B-3106 }{ }^{\mathrm{T}}\left(=\text { ATCC } 19769^{\mathrm{T}}=\text { CBS } 507.68^{\mathrm{T}}=\text { DSM } 40114^{\mathrm{T}}=\text { IFO } 12782^{\mathrm{T}}\right. \\
\left.=\text { JCM } 4331^{\mathrm{T}}=\mathrm{JCM} 4583^{\mathrm{T}}\right)\end{array}$ \\
\hline & "Streptomyces taitoensis" & NRRL B-16435 (= ATCC $29758=$ DSM $41499=$ IFO $13808=$ JCM 5008) \\
\hline \multirow{3}{*}{$\begin{array}{l}\text { Streptomyces salmonis (formerly } \\
\text { Streptoverticillium salmonis) }\end{array}$} & Streptomyces salmonis ${ }^{c}$ & NRRL B-1472 $\left(=\right.$ DSM $40895^{\mathrm{T}}=$ IFO $\left.15865^{\mathrm{T}}=\mathrm{JCM} 4083^{\mathrm{T}}\right)$ \\
\hline & Streptomyces aureoversile ${ }^{d}$ & $\begin{array}{l}\text { NRRL B-3325 }\left(=\text { ATCC } 15853^{\mathrm{T}}=\text { ATCC } 25433^{\mathrm{T}}=\text { CBS } 664.69^{\mathrm{T}}=\text { DSM }\right. \\
\left.40387^{\mathrm{T}}=\text { IFO } 13021^{\mathrm{T}}=\mathrm{JCM} 4457^{\mathrm{T}}\right)\end{array}$ \\
\hline & "Streptomyces reticulusruber" & NRRL B-1484 (= ATCC $25607=$ CBS $208.78=$ DSM $40893=$ NCIBM 12857) \\
\hline $\begin{array}{l}\text { Streptomyces ladakanum (formerly } \\
\text { Streptoverticillium ladakanum) }\end{array}$ & Streptomyces ladakanum ${ }^{c}$ & 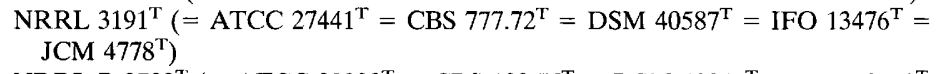 \\
\hline \multirow{2}{*}{$\begin{array}{l}\text { Streptomyces mobaraensis } \\
\text { (formerly Streptoverticillium } \\
\text { mobaraense) }\end{array}$} & Streptomyces mobaraensis ${ }^{c}$ & $\begin{aligned} \text { NRRL B-3729 } & =\text { ATCC } 29032^{\mathrm{T}}=\text { CBS } 199.75^{\mathrm{T}}=\text { DSM } 40847^{\mathrm{T}}=\text { IFO } 13819^{\mathrm{T}} \\
= & \left.\text { JCM } 4168^{\mathrm{T}}=\operatorname{NCIB} 11159^{\mathrm{T}}\right)\end{aligned}$ \\
\hline & "Streptomyces luteoreticuli" & $\begin{array}{l}\text { NRRL B- } 12435^{\mathrm{T}}\left(=\text { ATCC } 27446^{\mathrm{T}}=\text { CBS } 723.72^{\mathrm{T}}=\mathrm{DSM} 40509^{\mathrm{T}}=\mathrm{IFO}\right. \\
\left.13422^{\mathrm{T}}=\mathrm{JCM} 4788^{\mathrm{T}}\right)\end{array}$ \\
\hline
\end{tabular}

${ }^{a}$ See reference 7 .

${ }^{b}$ NRRL, Agricultural Research Service Culture Collection, National Center for Agricultural Utilization Research, Peoria, Ill.

c See reference 18.

"Former valid Streptoverticillium species or subspecies name not validated as a Streptomyces taxon by Witt and Stackebrandt (18). 


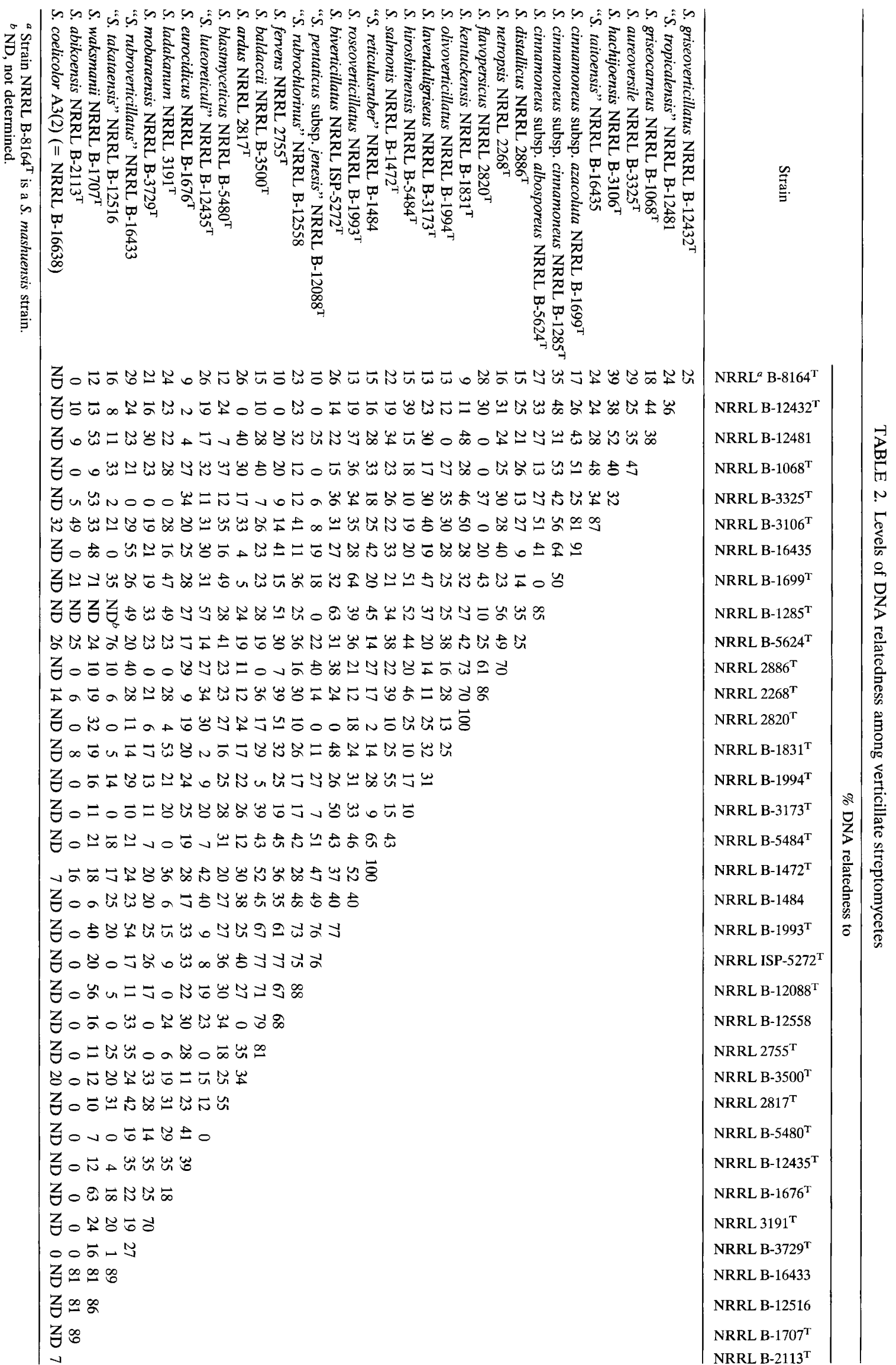




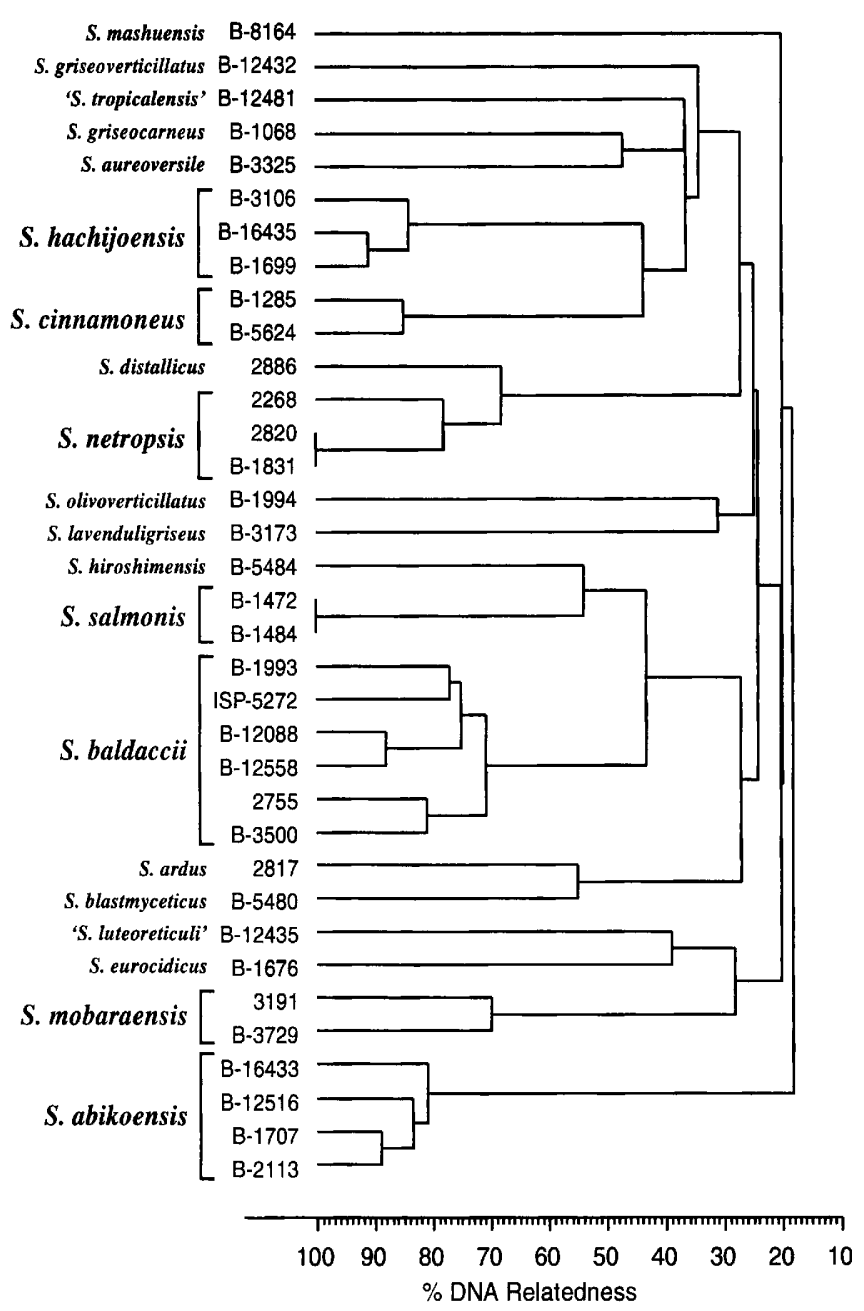

FIG. 1. Dendrogram resulting from average clustering of DNA relatedness matrix data. The species or species cluster names indicated on the left were defined at levels of DNA relatedness of $>70 \%$.

abikoensis cluster and generally low levels of relatedness (0 to $55 \%$ ) to all of the other strains included in this study.

Within the $S$. baldaccii cluster, Streptomyces biverticillatus NRRL ISP-5272 ${ }^{\mathrm{T}}$, Streptomyces fervens NRRL $2755^{\mathrm{T}}$, Streptomyces roseoverticillatus NRRL B-1993", and "Streptomyces nubrochlorinus" NRRL B-12558 can validly be considered subjective synonyms of $S$. baldaccii NRRL B-3500 ${ }^{\mathrm{T}}$ since the levels of DNA relatedness among these strains are more than $70 \%$. "Streptomyces pentaticus subsp. jenesis" NRRL B-12088 ${ }^{\mathrm{T}}$, a member of the Streptomyces griseocarneus cluster, also appears to be synonymous with $S$. baldaccii on the basis of levels of DNA homology of $>70 \%$ among all of the strains belonging to this DNA relatedness cluster.

Streptomyces netropsis NRRL $2268^{\mathrm{T}}$, Streptomyces kentuckensis NRRL B-1831 ${ }^{\mathrm{T}}$, and Streptomyces flavopersicus NRRL $2820^{\mathrm{T}}$ clustered phenotypically with $S$. baldaccii in the study of Locci and Schofield, but these species are clearly subjective synonyms of the distinct taxon $S$. netropsis on the basis of DNA homology data, as shown in Table 2 and Fig. 1. All three of these strains also exhibit low levels of DNA relatedness to $S$. baldaccii. Streptomyces distallicus NRRL $2886^{\mathrm{T}}$ could also be considered marginally synonymous with $S$. netropsis, since it exhibits 70 and $73 \%$ DNA relatedness with $S$. netropsis NRRL
$2268^{\mathrm{T}}$ and $S$. kentuckensis NRRL B-1831 ${ }^{\mathrm{T}}$, respectively, but reproducibly exhibits only $61 \%$ DNA relatedness with $S$. flavopersicus NRRL $2820^{\mathrm{T}}$, which causes it to join the main cluster at a homology level of $62 \%$. This could indicate that this strain is related to $S$. netropsis at the subspecies level.

Streptomyces griseoverticillatus NRRL B-12432 ${ }^{\mathrm{T}}$ and Streptomyces hiroshimensis NRRL B-5484 ${ }^{\mathrm{T}}$, the remaining species in the $S$. baldaccii phenotypic cluster, appear to be separate species on the basis of their low levels of DNA relatedness to all of the other species included in this study.

The Streptomyces cinnamoneus subsp. cinnamoneus phenotypic cluster disintegrated after the levels of DNA relatedness among its members were determined, since the only strains belonging to this phenotypic cluster that exhibited a species level DNA homology value $(85 \%)$ were type strains $S$. cinnamoneus subsp. cinnamoneus NRRL B-1285 and S. cinnamoneus subsp. albosporeus NRRL B-5624 (Table 2 and Fig. 1). Streptomyces blastmyceticus NRRL B-5480 ${ }^{\mathrm{T}}$, Streptomyces eurocidicus NRRL B-1676 ${ }^{\mathbf{T}}$, and Streptomyces lavenduligriseus NRRL B-3173 ${ }^{\mathrm{T}}$ appear to be distinct taxa, again on the basis of the low levels of DNA relatedness to all of the other strains studied.

The relationships among the strains in the remaining phenotypic clusters were also rearranged on the basis of the DNA relatedness data in Table 2 and Fig. 1. The members of the Streptomyces griseocarneus phenotypic cluster were separated and placed in five distinct taxa on the basis of low levels of DNA relatedness to the other strains included in this study (Streptomyces ardus NRRL $2817^{\mathrm{T}}$,S griseocarneus NRRL B-1068 ${ }^{\mathrm{T}}$, Streptomyces mashuensis NRRL B-8164 ${ }^{\mathrm{T}}$, Streptomyces olivoverticillatus NRRL B-1994", and "Streptomyces tropicalensis" NRRL B-12481).

Streptomyces cinnamoneus subsp. azacoluta NRRL B-1699 ${ }^{\mathrm{T}}$, a member of the $S$. griseocarneus cluster, and "Streptomyces taitoensis" NRRL B-16435 are subjective synonyms of Streptomyces hachijoensis NRRL B-3106 ${ }^{\mathrm{T}}$ (levels of DNA relatedness, $>83 \%$ ). Streptomyces mobaraensis NRRL B-3729 $9^{\mathrm{T}}$ and Streptomyces ladakanum NRRL $3191^{\mathrm{T}}$, the type strains of their respective phenotypic clusters, merge weakly at a level of DNA relatedness of $70 \%$ as synonyms of the species that has priority (i.e., S. mobaraensis), but "Streptomyces luteoreticul" NRRL B- $12435^{\mathrm{T}}$ appears to be a genetically distinct taxon. Streptomyces salmonis NRRL B-1472 ${ }^{\mathrm{T}}$ exhibited $100 \%$ DNA homology with "Streptomyces reticulusruber" NRRL B-1484, a strain in the Agricultural Research Service Culture Collection which is used as a test microorganism for discoloration of plastics. Streptomyces aureoversile NRRL B-3325 $5^{\mathrm{T}}$, a member of the $S$. salmonis phenotypic cluster, appears at this time to be a distinct taxon on the basis of its low levels of DNA relatedness to all other strains.

The low levels of DNA relatedness observed among unrelated strains are a reflection of the genus background level of chromosomal homology as determined spectrophotometrically. The levels of DNA relatedness of strains belonging to the major DNA relatedness clusters to a typical streptomycete, Streptomyces coelicolor A3(2) (= NRRL B-16638), range from 0 to $32 \%$ (Table 1 ).

The results of the analysis of levels of DNA relatedness among the verticillate strains included in this study clearly indicate that the species groups defined by Locci and Schofield (8), which were based on phenotypic numerical taxonomy data, should be reorganized to reflect the genetic relatedness of the strains. Valid descriptions of the reorganized species will require reevaluation of the morphological and physiological data collected in the previous numerical taxonomic study, as well as an examination of possible new physiological properties, to 
determine if differential characteristics which separate and clearly described the taxa defined by molecular systematic methods can be found.

\section{ACKNOWLEDGMENT}

The able technical assistance of E. N. Hockstra in the isolation and purification of DNA and in the performance of the DNA hybridization experiments required for this study is gratefully acknowledged.

\section{REFERENCES}

1. Baldacci, E. 1958. Development in the classification of actinomycetes. G. Microbiol. 6:10-27.

2. Gladek, A., M. Mordarski, M. Goodfellow, and S. T. Williams. 1985. Ribosomal ribonucleic acid similarities in the classification of Streptomyces. FEMS Microbiol. Lett. 26:175-180.

3. Kurtzman, C. P., M. J. Smiley, C. J. Johnson, L. J. Wickerham, and G. B. Fuson. 1980. Two new and closely related heterothallic species, Pichia amylophila and Pichia mississippiensis: characterization by hybridization and deoxyribonucleic acid reassociation. Int. J. Syst. Bacteriol. 30:208-216.

4. Labeda, D. P. 1993. DNA relatedness among strains of the Streptomyces lavendulae phenotypic cluster group. Int. J. Syst. Bacteriol. 43:822-825.

5. Labeda, D. P., and A. J. Lyons. 1991. Deoxyribonucleic acid relatedness among species of the "Streptomyces cyaneus" cluster. Syst. Appl. Microbiol. 14:158-164.

6. Labeda, D. P., and A. J. Lyons. 1991. The Streptomyces violaceusniger cluster is heterogeneous in DNA relatedness among strains: emendation of the descriptions of $S$. violaceusniger and Streptomyces hygroscopicus. Int. J. Syst. Bacteriol. 41:398-401.

7. Locci, R., J. Rogers, P. Sardi, and G. M. Schofield. 1981. A preliminary numerical study of named species of the genus Streptoverticillium. Ann. Microbiol. Enzimol. 31:115-121.
8. Locci, R., and G. M. Schofield. 1989. Genus Streptoverticillium Baldacci 1958, 15 , emend. mut. char. Baldacci, Farina and Locci $1966,168^{\mathrm{AL}}$, p. 2492-2504. In S. T. Williams, M. E. Sharpe, and J. G. Holt (ed.), Bergey's manual of systematic bacteriology, vol. 4. Williams \& Wilkins, Baltimore.

9. Maniatis, T., E. F. Fritsch, and J. Sambrook. 1982. Molecular cloning: a laboratory manual. Cold Spring Harbor Laboratory, Cold Spring Harbor, N.Y.

10. Marmur, J. 1961. A procedure for the isolation of deoxyribonucleic acid from microorganisms. J. Mol. Biol. 3:208-218.

11. Pridham, T. G. 1976. Identification of streptomycetes and streptoverticillia at the species level: revision of the 1965 system, p. 175-181. In T. Arai (ed.), The actinomycetes: the boundary microorganisms. Toppan Co. Ltd., Tokyo.

12. Pridham, T. G., and A. J. Lyons. 1980. Methodologies for Actinomycetales with special reference to streptomycetes and streptoverticillia, p. 153-224. In A. Dietz and D. W. Thayer (ed.), Actinomycete taxonomy. Special Publication no. 6. Society for Industrial Microbiology, Arlington, Va.

13. Seidler, R. J., M. D. Knittel, and C. Brown. 1975. Potential pathogens in the environment: cultural reactions and nucleic acid studies on Klebsiella pneumoniae from chemical and environmental sources. Appl. Microbiol. 29:819825 .

14. Seidler, R. J., and M. Mandel. 1971. Quantitative aspects of deoxyribonucleic acid renaturation: base composition, state of chromosome replication, and polynucleotide homologies. J. Bacteriol, 106:608-614.

15. Shirling, E. B., and D. Gottlieb. 1966. Methods for characterization of Streptomyces species. Int. J. Syst. Bacteriol. 16:313-340.

16. Toyama, H., M. Okanishi, and H. Umezawa. 1974. Heterogeneity among whorl-forming streptomycetes determined by DNA reassociation. J. Gen. Microbiol. 80:507-514

17. Williams, S. T., M. Goodfellow, G. Alderson, E. M. H. Wellington, P. H. A. Sneath, and M. J. Sackin. 1983. Numerical classification of Streptomyces and related genera. J. Gen. Microbiol. 129:1743-1813.

18. Witt, D., and E. Stackebrandt. 1990. Unification of the genera Streptoverticillium and Streptomyces, and amendation of Streptomyces Waksman and Henrici 1943, 339 AL. Syst. Appl. Microbiol. 13:361-371. 